\title{
SENTENCIA DE LA CORTE DE DISTRITO DE ESTADOS UNIDOS, DISTRITO SUR, NUEVA YORK, DEL 24 DE JUNIO DE I987: STEINBERG \\ V. COLUMBIA PICTURES INDUSTRIES, INC., 663 F. SUPP. 706 (S.D.N.Y. I987)
}

TRADUCCIÓN DE DANIEL MOLANO CAMACHO*

SENTENCIA Y RESOLUCIÓN JUDICIAL

Staton, Juez del Distrito.

En estas acciones incoadas por infracción a los derechos de autor, el demandante, Saul Steinberg, demanda a los productores, promotores, distribuidores y publicistas de la película "Moscow on the Hudson" ("Moscow"). Steinberg es un artista cuyo reconocimiento se debe, en parte, a las caricaturas e ilustraciones por él realizadas para la revista The New Yorker. El demandado, Columbia Pictures Industries, Inc. (Columbia), por el otro lado, está en el negocio de la producción, promoción y distribución de películas cinematográficas, dentro de las cuales se incluye "Moscow". El también demandado RCA Corporation (RCA) se encuentra relacionado con Columbia, en la medida que se encargó de promocionar y distribuir la versión en formato casero de "Moscow", al igual que el demandado Diener Hauser Bates Co. (DHB), quien fungió como agente de publicidad de "Moscow". Los demás demandados fueron vinculados a la reclamación por medio de una providencia de esta Corte fechada el 17 de noviembre de 1986. Estos demandantes, entonces, se dividen en dos categorías: (1) los afiliados a Columbia y RCA que estuvieron relacionados con la distribución de "Moscow" en Estados Unidos y en el exterior, y (2) los propietarios de los principales periódicos que publicaron la publicidad presuntamente infractora.

Los demandados de la segunda acción indicada son joint ventures asociados con Columbia, o bien periódicos que publicaron la publicidad presuntamente

* Estudiante de quinto año de Derecho de la Universidad Externado de Colombia, promoción 2018, y monitor del Departamento de Derecho Procesal de la misma universidad. Bogotá (Colombia). Para citar el texto: "Sentencia de la Corte de Distrito de Estados Unidos, Distrito Sur, Nueva York, del 24 de junio de 1987: Steinberg v. Columbia Pictures Industries, Inc., 663 F. Supp. 706 (S.D.N.Y. 1987)”, Revista La Propiedad Inmaterial n. ${ }^{\circ}$ 25, Universidad Externado de Colombia, enero-junio 2018, pp. 155-167. DOI: https://doi.org/10.18601/16571959.n25.08 
infractora de "Moscow". Esta acción se consolidó con la primera estipulación, de fecha 3 de abril de 1987.

El demandante alega que el póster promocional de "Moscow" publicado por los demandados infringe sus derechos de autor sobre la ilustración dibujada por él para la portada del 29 de marzo de 1976 de la revista The New Yorker, en violación del 17 U.S.C. $\$ \$ 101-810$. Los demandados niegan tal afirmación, y sostienen su defensa sobre los fundamentos del fair use alegando la existencia de pariodia, estoppel y caducidad.

I.

Para conceder el juicio sumario, Fed. R. Civ.P.56 se requiere que la corte encuentre que "no hay una genuina controversia relativa a los aspectos de hecho, y que por ello la parte accionante tiene la prerrogativa de un juicio de derecho". Para alcanzar su decisión, la corte debe "evaluar si hay o no aspectos de hecho pendientes de probar, mientras resuelve ambigüedades y establece inferencias razonables contra la parte actora”. Knight v. U.S. Fire Inc. Co., 804 F.2d 9, 11 (2d Cir. 1986), citando Anderson v. Liberty Lobby, 477 U.S. 242, 106 S.Ct. 2505, 2509-11, 91 L.Ed.2d 202 (1986).

El juicio sumario es frecuentemente desfavorable en los casos de derechos de autor, y las cortes generalmente se muestran reacias a realizar comparaciones y determinaciones subjetivas a tal propósito. Hoehling v. Universal City Studios, Inc., 618 F.2d 972, 977 (2d Cir. 1980), citando Arnstein v. Porter, 154 F.2d 464, 474 (2d Cir. 1946). Sin embargo, recientemente, este circuito ha "reconocido que la corte puede determinar la inexistencia de una infracción como un aspecto de derecho dentro de una solicitud de juicio sumario. Warner Brothers v. American Broadcasting Cos., 720 F.2d 231, 240 (2d Cir. 1983), citando Durham Industries, Inc. v. Tomy Corp., 630 F.2d 905, 918 (2d Cir. 1980). Ver también Hoehling, 618 F.2d at 977; Walker v. Time-Life Films, Inc., 615 F.Supp. 430, 434 (S.D.N.Y. 1985), aff'd, 784 F.2d 44 (2d Cir. 1986), cert. denied, __ U.S. _ , 106 S.Ct. 2278, 90 L.Ed.2d 721 (1986). "Cuando la evidencia es tan sobrecogedora que se justificaría que la corte profiriera un veredicto directo en el proceso, es apropiado conceder el juicio sumario. Silverman v. CBS Inc., 632 F.Supp. 1344, 1352 (S.D.N.Y. 1986) (concediendo juicio sumario al demandado en la contrademanda o demanda de reconvención por la infracción de los derechos de autor).

Las voluminosas entregas que acompañaron este cruce de mociones no dejan aspectos de hecho por demostrar a través de pruebas que puedan allegarse con posterioridad al proceso. Además, las determinaciones fácticas necesarias para tomar esta decisión no involucran conflictos testimoniales que hagan depender la resolución de la evaluación sobre la credibilidad del testigo. Adicional a ello, este caso difiere de la mayoría de las controversias por acciones de infracción a los derechos de autor, en las cuales es preferible dejar la determinación del problema 
al jurado: en este evento, cada parte ha manifestado que su caso está completo siguiendo el proceso por juicio sumario, y como ninguna de ellas ha solicitado un jurado, la Corte será quien determine los hechos en el juicio. Finalmente, los intereses de economía procesal coadyuvan a que este caso sea resuelto en la presente etapa. Por ello, el juicio sumario es apropiado.

II.

Los hechos esenciales no son discutidos por las partes a pesar de sus desacuerdos frente a asuntos no esenciales. El 29 de marzo de 1976, The New Yorker publicó como ilustración de portada el trabajo que es objeto de la presente demanda, imprimiendo en el mismo la ampliamente conocida forma paródica de ver el mundo por los "New Yorker's". La revista registró la ilustración en la Oficina de Derechos de Autor de Estados Unidos (United States Copyright Office) y seguidamente asignó los derechos de autor a Steinberg. Aproximadamente tres meses después, el demandante y The New Yorker realizaron un acuerdo para imprimir y vender un número determinado de posters de la ilustración de portada.

Es indiscutible que las duplicaciones (o copias) no autorizadas del póster fueron realizadas por personas desconocidas, aun cuando las partes estuvieron en desacuerdo frente a la existencia de acciones para prevenir la distribución de las falsificaciones por el demandante. El demandante también reconoció que otro número de posters fueron creados y publicados representando otras localidades en la misma forma en que él representó a Nueva York en su ilustración. Estos hechos, sin embargo, son irrelevantes para los propósitos de este caso, en donde interesa únicamente la relación entre las ilustraciones del demandante y las del demandado.

La ilustración de los demandados fue creada para promocionar la película "Moscow on the Hudson", que cuenta las aventuras de un moscovita que llega a domiciliarse en Nueva York. Para el diseño de la ilustración, el director de arte de Columbia, Kevin Nolan, admitió específicamente haber tomado como referencia el póster de Steinberg, póster que en efecto había comprado y colgado en su oficina, junto con otros. Aunado a ello, Nolan explícitamente dio instrucciones al artista externo con quien contactó para la ejecución de su diseño, Craig Nelson, para que usara el póster de Steinberg con el fin alcanzar una imagen más reconocible de Nueva York. En efecto, Nelson admitió haber usado la fachada de un edificio en particular, en específico luego de que Nolan le hubiera sugerido que ello le daría un estilo más "New York-ish" a su dibujo. Curtis Affidavit 28(c). Si bien los edificios no son idénticos, son tan similares que es imposible, específicamente desde el punto de vista del artista en su testimonio, no encontrar que los demandados imprevisiblemente copiaron al demandante ${ }^{1}$.

1 Nolan también alegó haberse inspirado en algunos de los posters que fueron inspirados por Steinberg; sin embargo, tal inspiración segundaria resulta irrelevante para determinar si el póster de "Moscow" infringe o no los derechos de autor del demandado al haber sido copiados sin permiso alguno. 
Para resolver el problema de la infracción es necesario considerar los posters en sí mismos. La ilustración de Steinberg presenta una vista de "ojo de pájaro" a través de una parte del borde occidental de Manhattan, pasando por el río Hudson, y presentando una versión telescópica del resto de Estados Unidos y el Océano Pacífico, hasta una línea roja en el horizonte, después de la cual se hallan tres terrenos planos etiquetados como China, Japón y Rusia. El nombre de la revista, en la fuente tipográfica usual de The New Yorker, ocupa la quinta parte superior del póster, debajo de una suerte de línea azul que representa al cielo estilizado.

Las partes del póster que están más allá de Nueva York están minimizadas, para simbolizar la miope concepción de la centralidad en el mundo que tienen los neoyorquinos respecto de su ciudad. El resto de Estados Unidos ubicado al occidente del río Hudson, por ejemplo, es reducido a una línea marrón etiquetada con la vocablo "Jersey", junto con un trapecio verde claro y unos afloramientos de rocas rudimentarias, acompañados de los nombres de solo siete ciudades y dos estados diseminados sobre la superficie. Las pocas cuadras de Manhattan, por el contrario, son representadas con color y detalle. Las cuatro cuadras cuadradas de la ciudad que ocupan por completo la mitad del póster ubicada en la parte baja, incluyen la representación de numerosos edificios, peatones y carros, así como estacionamientos y postes de luz, con torres de agua en la parte superior de algunos de los edificios. El estilo tipo boceto del dibujo, así como la puntiaguda caligrafía de la letra, son características de Steinberg.

La ilustración de "Moscow" representa en el tercio inferior del póster a los tres personajes principales de la película superpuestos sobre una vista de "ojo de pájaro" de la ciudad de Nueva York, y continúa por el este a través de Manhattan y el Océano Atlántico, pasando por una rudimentaria representación de Europa, hasta llegar a un grupo de edificios de estilo ruso en el horizonte, etiquetados como "Moscow". Los créditos de la película aparecen sobre la parte baja de los personajes. La parte central del póster representa aproximadamente cuatro cuadras de la ciudad de Nueva York, con edificios bastante detallados, peatones y vehículos, un estacionamiento, y algunos tanques de agua y postes de luz. El artista de Columbia añadió unos cuantos puntos de referencia de Nueva York aparentemente al azar en su ilustración, al parecer para hacer la locación más fácilmente reconocible. Detrás de la franja de color azul etiquetada como "Océano Atlántico", Europa es representada por Londres, París y Roma, cada una etiquetada con un solo símbolo representativo de las mismas (aun cuando el símbolo usado para representar a Roma es la Torre de Pisa).

El horizonte detrás de Moscú está delineado por una línea de crayón rojo, por encima de la cual se encuentra el título de la película y una breve introducción escrita de la trama de la misma. El póster es iniciado por una fina línea azul, aparentemente para representar el cielo. Este póster es ejecutado en una combinación de estilos: los tres personajes, cuyas similitudes fueron copiadas de una fotografía, tienen rostros reales y unas ropas poco detalladas, y los edificios de la ciudad son 
dibujados con mucho detalle pero con algo de "estilo tipo boceto". La letra del dibujo es puntiaguda y hecha a mano, y se encuentra puesta en bloques y en mayúsculas de manera sustancialmente idéntica a la del demandante, mientras que los textos impresos en la parte superior e inferior del poster están relacionados en la fuente tipográfica usual asociada con la revista The New Yorker².

III.

Para acceder a una acción por infracción a los derechos de autor, el demandante tiene que probar su propiedad sobre los mismos y la existencia de la copia por parte del demandado. Reyher v. Children's Television Workshop, 533 F.2d 87, 90 (2d Cir. 1976); Durham Industries, 630 F.2d at 911; Novelty Textile Mills, Inc. v. Joan Fabrics Corp., 558 F.2d 1090, 1092 (2d Cir. 1977). No hay discusión sustancial alguna relacionada con la titularidad del demandante sobre los derechos de autor de su ilustración. Por lo cual, en orden a triunfar en el juicio de responsabilidad, el demandante tiene que establecer el segundo elemento de la causa de la acción.

"Debido a la dificultad inherente de obtener evidencia directa del copiado, usualmente el mismo se prueba por medio de evidencia circunstancial de acceso al trabajo protegido por derechos de autor y por las similitudes sustanciales que constituyan material protegible en los dos trabajos". Reyher, 533 F.2d at 90, citando Arnstein v. Porter, 154 F.2d 464, 468 (2d Cir. 1946). Ver también Novelty Textile Mills, 558 F.2d at 1092. "Por supuesto, si no hay similitudes, ninguna cantidad de evidencia de acceso será suficiente para probar la copia". Arnstein v. Porter, 154 F.2d at 468. Véase también Novelty Textile Mills, 558 F.2d at 1092 n. 2.

El acceso del demandado a la ilustración del demandante está establecido más allá de toda casualidad. Por ello, el único problema restante atinente a la responsabilidad es la determinación de si hay una similitud sustancial entre la obra protegida por derechos de autor y el trabajo acusado, para así establecer si hay o no violación de tales derechos del demandante. El problema central de la "similitud sustancial", que puede ser considerada muy cercana a una cuestión de hecho, puede a su vez ser válidamente decidido como una cuestión de derecho.

"Similitud sustancial" es un concepto evasivo. Este circuito ha reconocido recientemente que:

La "similitud sustancial" que soporta una inferencia de copiado suficiente para establecer infracción de derechos de autor no es un concepto familiar para el grueso del público. Es un término usado en la Corte, para atacar un delicado balance entre la protección de la que son titulares los autores bajo una ley del Congreso y la libertad que existe en otros para crear sus propios trabajos por fuera del área de protegida por infracción.

2 La fuente tipográfica no es objeto de protección por los derechos de autor, pero la similitud refuerza la impresión de que los demandados copiaron la ilustración del demandante. 
Warner Bros., 720 F.2d at 245.

La definición de "similitud sustancial" en este circuito se da en el supuesto en el que "un observador lego pueda reconocer que el trabajo alegado como copia ha sido apropiado de la obra protegida por derechos de autor". Ideal Toy Corp. v. Fab-Lu Ltd., 360 F.2d 1021, 1022 (2d Cir. 1966); Silverman v. CBS, Inc., 632 F.Supp. at 1351-52. El demandante no necesita volver a usar el riguroso test del "observador ordinario" establecido por el Juez Learned Hand en Peter Pan Fabrics, Inc. v. Martin Weiner Corp., 274 F.2d 487 (2d Cir. 1960). Uneeda Doll Co., Inc. v. Regent Baby Products Corp., 355 F.Supp. 438, 450 (E.D.N.Y. 1972). Bajo la fórmula del Juez Hand, habría similitud sustancial solo cuando "el observador ordinario, a menos que se detenga a detectar las disparidades, estuviera dispuesto a pasar por alto las mismas, y considerara el valor estético como igual". 274 F.2d at 489.

Con todo, hoy en día se reconoce que "el copiado no tiene que recaer sobre cada detalle de la obra, sino que basta con que la copia sea sustancialmente similar a la obra protegida por derechos de autor". Comptone Co. v. Rayex Corp., 251 F.2d 487, 488 (2d Cir. 1958). Véase también Durham Industries, 630 F.2d at 911-12; Novelty Textile Mills, 558 F.2d at 1092-93.

En la determinación sobre la existencia de similitud sustancial entre los dos trabajos es crucial distinguir entre una idea y su expresión. Es un axioma de la ley de derechos de autor, establecido en jurisprudencia y también codificado en el 17 U.S.C. $\$ 102(b)$, que únicamente la expresión particular de una idea es protegible, mientras que la idea por sí sola no lo es. Ver, e.g., Durham Industries, 630 F.2d at 912; Reyher, 533 F.2d at 90, citando Mazer v. Stein, 347 U.S. 201, 217, 74 S.Ct. 460, 470, 98 L.Ed. 630 (1954); Baker v. Selden, 101 U.S. (11 Otto) 99, 25 L.Ed. 841 (1879). Ver también Warner Bros., 720 F.2d at 239.

"La distinción idea/expresión, aun cuando es una herramienta imprecisa, no ha sido abandonada debido a que, al día de hoy, no se ha descubierto una mejor forma de reconciliar la competencia que se presenta entre los dos intereses sociales que proveen la justificación de la concesión y las restricciones de protección en derechos de autor", a saber, ambos recompensan el ingenio individual, y sin embargo, permiten el progreso y el mejoramiento basado en el mismo tema por parte de otros sujetos diferentes al autor. Durham Industries, 630 F.2d at 912, citando Reyher, 533 F.2d at 90.

No se discute que los demandados no pueden ser declarados responsables por el uso de la idea de realizar un mapa del mundo desde una perspectiva miope y egocéntrica. No se ha desarrollado ningún principio rígido, sin embargo, para cerciorarse de cuándo alguien ha ido más allá de la idea y ha llegado a la expresión, "la decisión debe ser entonces inevitablemente ad hoc". Peter Pan Fabrics, Inc. v. Martin Weiner Corp., 274 F.2d 487, 489 (2d Cir. 1960) (L. Hand, J.). Como el Juez Frankel consideró alguna vez, "los ojos de Dios y el sentido común pueden ser útiles para un estudio profundo de casos reportados y no reportados, que por 
sí mismos se encuentran atados a hechos muy particulares". Couleur International Ltd. v. Opulent Fabrics, Inc., 330 F.Supp. 152, 153 (s.D.N.Y. 1971).

Incluso a primera vista, uno puede ver la chocante relación estilística entre los posters, y puesto que uno de los ingredientes del estilo es la expresión, esta relación es significativa. La ilustración de los demandados fue realizada en el "sketchy" y descomplicado estilo que se ha convertido en uno de los sellos característicos de Steinberg. Ambos ilustradores representaron una vista de "ojo de pájaro" desde el borde de Manhattan y el río que lo bordea, hasta el resto del mundo. Ambos representaron aproximadamente cuatro cuadras de la ciudad en detalle, y se tornaron crecientemente minimalistas en el diseño en la medida en que se iban adentrando al fondo de la imagen. Ambos utilizaron una estrecha línea azul a través de la parte superior del póster para representar el cielo, y ambos delinearon el horizonte con una línea o banda horizontal de rojo primario ${ }^{3}$.

La similitud más fuerte se evidencia en la representación de la cuadras de Nueva York. Ambos artistas escogieron un punto privilegiado que viera directamente hacia una calle abierta de doble sentido, que es intersectada por dos avenidas antes de llegar al río. A pesar de las alegaciones de la demandada, tal presentación de la ciudad no es una forma inevitable de representar los bloques en una ciudad en bloques, en especial si se tiene que la mayoría de calles en Nueva York son de un solo sentido. Si inclusive un fotógrafo podría ser sujeto de protección por el derecho de autor, en la medida en que "ninguna foto, sin importar lo simple, puede ser despojada de la influencia personal impresa por el autor en ella" (Time Inc. v. Bernard Geis Assoc., 293 F.Supp. 130, 141 [s.D.N.Y.1968], citando Bleistein, supra), difícilmente se podría negar el derecho de un artista de proteger su perspectiva y diseño en un dibujo, especialmente en consideración al concepto general y los detalles individuales del mismo. Efectivamente, el hecho de que los demandados cambiaran el nombre de las calles mientras mantenían la misma representación gráfica debilita su caso: si hubieran realmente intentado representar de manera realística las calles etiquetadas en el póster, sus cuatro cuadran no habrían resultado tan estrechamente similares a las cuatro cuadras representadas por el demandante. Por otra parte, el hecho de que hubieran mezclado los puntos representativos de la ciudad para simbolizar la confusión del protagonista moscovita en la nueva ciudad, como argumentaron en su momento, no retrae las similitudes existentes entre su póster y el de Steinberg.

3 Los demandantes alegan que puesto que el uso de la banda primaria de colores corresponde a una técnica tradicional japonesa, su adopción o utilización no puede infringir los derechos de Steinberg. Sin embargo, tal argumento ignora el principio según el cual, mientras "[o]tros son libres de copiar el original [...] [e]stos no son libres de copiar la copia”. Bleistein v. Donaldson Lithographing Co., 188 U.S. 239, 250, 23 S.Ct. 298, 300, 47 L.Ed. 460 (1903) (Holmes, J.). Cfr. Dave Grossman Designs, Inc. v. Bortin, 347 F.Supp. 1150, 1156-57 (N.D.Ill. 1972) (un artista puede usar el mismo objeto y estilo que otro "siempre que el segundo artista no copie sustancialmente la expresión específica de la idea del [primer artista]”). 
Si bien no todos los detalles son idénticos, muchos de ellos podrían ser confundidos uno por otro; por ejemplo, la representación de las torres de agua, los carros, la señal roja por encima del estacionamiento, e incluso muchos de los edificios individuales. Las formas, ventanas y la configuración de varios edificios es sustancialmente similar. Los ornamentos, fachadas y detalles de los edificios de Steinberg aparecen también en el póster del demandado, aun cuando lo hacen ocasionalmente en diferentes ubicaciones. En este contexto, es significativo el hecho de que Steinberg no hubiera representado ningún edificio realmente erigido en Nueva York; por el contrario, se inspiró en la apariencia general de las estructuras del costado occidental de Manhattan para crear su propia versión del estilo New York-ish de las estructuras. Así, las similitudes entre los edificios representados en "Moscow" y en el póster de Steinberg no pueden ser explicadas cerciorándose de que ambos artistas hubieran elegido los mismos edificios para sus ilustraciones. Por ello, la similitud únicamente puede ser explicada en la medida en que el demandado copió el trabajo del demandante. Similarmente, las locaciones y los tamaños, así como los errores y anomalías de los sombreados y las luces de las calles de Steinberg, son meticulosamente copiados.

Adicional a ello, el artista de Columbia usó en el poster de "Moscow" el infantil y puntiagudo estilo de impresión que se ha convertido en uno de los sellos distintivos de Steinberg para marcar los nombres de las calles, lo cual solo se puede explicar en cuanto es una copia. No hay una justificación inherente para realizar el uso de tal estilo de letra para etiquetar las calles de la ciudad de Nueva York, pues tal estilo de letra solo se encuentra asociado a Nueva York a través de Steinberg.

Mientras el póster de los demandados muestran la ciudad de Moscú en el horizonte con mucho más detalle que cualquier otra representación realizada por el demandante en la parte de atrás de su ilustración, este hecho por sí solo no tiene la vocación de cambiar la conclusión. La "similitud sustancial" no exige identidad, y la "duplicación o identidad no es necesaria para establecer una infracción". Krofft, 562 F.2d at 1167. Ni la representación de Moscú, ni la perspectiva desde oriente, ni la presencia de puntos de referencia distribuidos al azar en la ciudad de Nueva York son suficientes para eliminar la similitud sustancial entre los posters. Como el Juez Hand escribió, "ningún plagiador podrá excusar su infracción mostrando cuánto de su trabajo no pirateó". Sheldon v. Metro-Goldwyn Pictures Corp., 81 F.2d 49, 56 (2d Cir.), cert. denied, 298 U.S. 669, 56 S.Ct. 835, 80 L.Ed. 1392 (1936).

Los demandados alegaron que su póster no podría infringir los derechos de autor del demandante, porque solo una pequeña parte de los diseños podrían ser considerados similares. Tal argumento es factual y legalmente carente de mérito. "La infracción a derechos de autor puede ocurrir por razón de una similitud sustancial que solo involucre una pequeña parte de cada trabajo". Burroughs $v$. Metro-Goldwyn-Mayer, Inc., 683 F.2d 610, 624 n. 14 (2d Cir. 1982). Además, este caso involucra la totalidad del trabajo protegido y una parte iconográfica, en proporción, a una porción significativa del trabajo acusado como infractor. Cfr. 
Mattel, Inc. v. Azrak-Hamway Intern., Inc., 724 F.2d 357, 360 (2d Cir. 1983); Elsmere Music, Inc. v. National Broadcasting Co., 482 F.Supp. 741, 744 (s.D.N.Y.), aff'd, 623 F.2d 252 (2d Cir. 1980) (tomar una pequeña parte del trabajo puede violar los derechos de autor).

El proceso por medio del cual el póster de los demandados fue creado soporta el argumento. El "mapa", que es la parte sobre la cual el demandante está presentando su reclamo, fue diseñado de manera separada del resto del póster. La semejanza de los tres personajes principales, quienes fueron copiados de una fotografía, y los bloques de texto fueron superpuestos en el mapa ya completado individualmente. Declaración de Nelson en 21-22; Declaración de Nolan en 28.

También rechazo el argumento de los demandados según el cual cualquier similitud entre los trabajos configura un supuesto no protegible de scenes a faire, o "incidentes, caracteres o configuraciones que, como un asunto práctico, son indispensables o son estándar para el tratamiento de un tema determinado". Walker, 615 F.Supp. at 436. Ver también Reyher, 533 F.2d at 92. Es innegable que al dibujar las cuadras de la ciudad de Nueva York se esperen en el mismo edificios, peatones, vehículos, postes de luz y torres de agua. Sin embargo, la queja del demandante no recae sobre el mero uso de esos elementos en el póster; sino que reclama que los demandados copiaron su expresión de esos elementos en la escena de la calle.

Mientras que la evidencia de una creación independiente por parte de los demandados habría rebatido el caso prima facie del demandante, "la ausencia de cualquier evidencia compensatoria que pruebe una creación independiente de la fuente de la obra protegida puede dar lugar a una conclusión claramente errónea de que no hubo copia”. Roth Greeting Cards v. United Card Co., 429 F.2d 1106, 1110 (9th Cir. 1970). Ver también Novelty Textile Mills, 558 F.2d at 1092 n. 2.

Además, se reconoce de manera general que "desde que es exigido un nivel alto de similitud para prescindir de la prueba de acceso, lógicamente se sigue que en aquellos casos en donde la prueba de acceso es concedida, el nivel de similitud requerido será algo menor de aquel que hubiere sido exigido en ausencia de dicha prueba". 2 Nimmer $\$ 143.4$ at 634, citado en Krofft, 562 F.2d at 1172. Como los demandados tuvieron acceso a la imagen del demandante protegida por derechos de autor, un grado de similitud inferior de aquel que hubiere sido requerido sin tal prueba resulta suficiente para establecer la infracción a los derechos de autor. Sin embargo, en este caso las similitudes demostrables son tantas que la prueba de acceso, aun cuando se concedió, no habría sido necesaria para determinar la infracción.

IV.

Encuentro sin fundamento la aseveración de los demandados en el sentido de indicar que el póster de "Moscow" es evocativo de Steinberg, y que, por tratarse de una evocación, la misma se encuentra justificada bajo la modalidad de parodia 
dentro la doctrina "fair use", codificada en 17 u.s.c. $\$ 107$. Como lo ha sostenido este circuito, el trabajo protegido por derechos de autor debe ser "al menos en parte objeto de parodia". En el expediente no existe constancia alguna que establezca que el demandado intentaba satirizar la ilustración del demandado; y por el contrario, la declaración testimonial del director ejecutivo de arte de Columbia dirige a contradecir tal reclamación. Además, la aseveración según la cual los demandados conscientemente parodiaron la idea de una perspectiva paródica del mundo es inmaterial: las ideas no se protegen por el régimen de derechos de autor, y la infracción alegada por Steinberg es acerca de la expresión particular de esa idea. La variación de los demandados sobre la broma visual de la ilustración del demandante, sin un elemento de humor dirigido a un aspecto de la ilustración misma, no convierte la obra en una parodia, esto es, una actividad prevista como fair use de la obra del demandante.

Al codificar la regla jurisprudencial (case law) para determinar si un trabajo constituye un uso permitido (fair use) de otro, el Congreso instruyó a las cortes para que fueran considerados ciertos factores, el primero de los cuales indaga si el uso del trabajo presuntamente infractor de los derechos de autor es "de naturaleza comercial o si es utilizado para propósitos no lucrativos o educativos". 17 u.s.C. $\$ 107(1)$. Como indicó el Segundo Circuito en un contexto artístico diferente, "No estamos preparados para sostener que un [artista] comercial pueda plagiar un [...] [trabajo] protegido por derechos de autor, sustituir [algunos elementos] por su propia mano, [producir] una ganancia comercial, y luego eximirse de responsabilidad aduciendo que el resultado final de tal actuar constituye una parodia o sátira a la luz de la costumbre de la sociedad". MCA, Inc., 677 F.2d at 185.

$\mathrm{Al}$ analizar la naturaleza comercial o no comercial del póster de "Moscow" es útil diferenciar dos situaciones conceptuales diferentes: el material publicitario que promueve una parodia de un trabajo protegido por derechos de autor, y el material publicitado que por sí mismo infringe los derechos de autor. En el primer caso, el uso de elementos del trabajo protegido por derechos de autor en el material publicitario no necesariamente implica la infracción a los mismos, siempre y cuando el producto que es publicitado constituya un uso permitido (fair use) del trabajo protegido. Ver, e.g., Warner Bros., 720 F.2d at 242-44 (La transmisión promocional de series en televisión parodiando legalmente al personaje del cómic de Superman no infinge los derechos de autor sobre el personaje de Superman).

En el segundo caso, el trabajo publicitado no guarda relación con el trabajo protegido por derechos de autor, pero la publicidad en sí misma infringe las normas de tales derechos. En tal caso, los titulares de los derechos de autor pueden prevenir que la publicidad sea utilizada. Como lo indicó el Segundo Circuito, "sin importar qué tan conocida se vuelva una frase protegida por derechos de autor, el autor conserva la facultad de proteger su trabajo en contra de la apropiación del mismo para la promoción de ventas de productos comerciales". Warner Bros., 720 F.2d at 242. Ver, e.g., D.C. Comics, Inc. v. Crazy Eddie, Inc., 205 u.s.P.Q. 1177 
(s.D.N.y. 1979) (el descuento en cadenas electrónicas no permite publicitar en sus tiendas los productos usando una parodia de líneas comúnmente conocidas y asociadas al personaje de Superman protegido por derechos de autor).

La situación actual encaja en el segundo de los casos. Ni la película ni el póster de "Moscow" fueron concebidos para ser una parodia de la ilustración de Steinberg. El póster apenas toma prestados numerosos elementos de la ilustración de Steinberg para crear una pauta publicitaria atractiva para promocionar un producto comercial no relacionado con la obra original, esto es, la película. No hay una parodia de la ilustración involucrada, y los demandados no se encuentran bajo la protección por parodia concebida en la doctrina del uso permitido (fair use).

Los demás factores ordenados por el 17 U.s.C. $\$ 107$ no atenúan esta determinación. El trabajo protegido por derechos de autor objeto de la controversia constituye una creación artística 17 U.s.c. $\$ 107(2)$, que fue apropiada en una porción sustancial significativa por el trabajo del demandado. 17 U.s.c. $\$ 107(3)$. Así, en la determinación del valor del trabajo protegido por derechos de autor 17 U.s.c. $\$ 107(4)$, el demandante rindió testimonio ante la corte, para mostrar que su reputación se había visto perjudicada al hacer creer al púbico que él, voluntariamente, había prestado su trabajo a una empresa lucrativa.

V.

En su memorial, los demandados alegaron como defensas afirmativas estoppel y caducidad. Aun cuando Fed.R.Civ.P. 8(c) generalmente se requiere que las defensas afirmativas sean alegadas, las cortes han sido más indulgentes al respecto en los juicios sumarios. "Siendo inexistente el perjuicio del demandante, el demandado puede elevar una defensa afirmativa dentro de una petición de juicio sumario por primera vez". Rivera v. Anaya, 726 F.2d 564, 566 (9th Cir. 1984). Ver 2A, 6 J. Moore, Moorés Federal Practice 99 8.28, 56.02[2], 56.17[4] (2d ed. 1986). Es por ello apropiado para esta corte considerar tales defensas meritorias.

Los demandados basan las afirmaciones de sus defensas equitativas en las siguientes reclamaciones de hecho: (1) que se presume del demandante "inacción deliberada" por el periodo de ocho años frente a las numerosas falsificaciones de su póster y las adaptaciones de su idea que fueron realizadas en varias localidades, (2) que se presume falla del demandante para actuar en respuesta a la publicidad que apareció en los periódicos para promocionar "Moscow"; y (3) la afirmación de los demandantes según la cual Steniberg esperó seis meses antes de siquiera quejarse frente a Columbia sobre el uso del presuntamente trabajo infractor de su póster protegido por derechos de autor, circunstancia esta que a juicio de los demandantes constituyó una táctica del demandante para maximizar los damages que esperaba recibir.

En el expediente, sin embargo, no se encuentra fundamento para apoyar lo expuesto por los demandados. En primer lugar, Steinberg específicamente solicitó 
que la revista The New Yorker identificara las fuentes de falsificación de posters y su distribución continuada. En cuando a las llamadas adaptaciones de las ideas de Steinberg, no se encuentra evidencia que permita concluir que hubo una violación a los derechos de autor del creador, o siquiera que alguien lo creyera de tal manera. Como libremente lo reconoció el demandante, los derechos de autor no protegen las ideas, sino solo la expresión específica de una idea. Los ejemplos utilizados por el demandado para soportar su defensa no pueden ser considerados derivados de la idea de Steinberg; ninguno de ellos es una copia cercana al póster, mientras que el del demandado sí lo es. Finalmente, las dos últimas afirmaciones del demandado son refutadas por el bagaje probatorio, en la medida en que la evidencia mostró que The New Yorker escribió al New York Times en nombre del demandante una protesta apenas "Moscow" se estrenó, la cual fue conocida por Columbia tan solo algunas semanas después.

Por lo demás, aun cuando las afirmaciones fácticas de los demandados obraren como probadas en el expediente, lo cierto es que sus defensas equitativas habrían sido igualmente rechazadas pues no fueron establecidos los elementos necesarios para reconocer el estoppel y la caducidad.

"La parte que busque invocar la doctrina del estoppel debe solicitar y probar cada uno de los elementos esenciales de la misma, esto es: (1) una representación de hecho [...]; (2) una confianza legítima; y (3) un daño o perjuicio [...] resultante de la negación de la parte que lleva a cabo la representación”. Galvez v. Local 804 Welfare Trust Fund, 543 F.Supp. 316, 317 (E.D.N.Y. 1982), citando Haeberle v. Board of Trustees, 624 F.2d 1132 (2d Cir. 1980).

Los demandados no han establecido tan siquiera el primero de aquellos elementos. Argumentan que el presunto silencio del demandante durante el curso de la campaña publicitaria constituyó una representación suficiente de su aquiescencia, que configuró el primer requerimiento señalado en la doctrina. Sin embargo, como se indicó con anterioridad, el demandante no guardó silencio y, por el contrario, el expediente muestra que los demandados, a pesar de haber conocido las objeciones del demandante, continuaron promocionando la película con la misma publicidad, a la cual se sumó subsecuentemente el estreno de la versión en videocasete de "Moscow" que usó el mismo diseño promocional. Ver Lottie Joplin Thomas Trust v. Crown Publishers, 592 F.2d 651, 655 (2d Cir. 1978) (la defensa de estoppel cae siempre que el demandado falle en producir cualquier evidencia de detrimento dependiente de la presunta representación del demandante). Los demandados pasaron por alto, además, que el silencio o inacción, en ausencia de cualquier deber o relación entre las partes, no da lugar a elevar el estoppel. Whiting Corp. v. Home Inc. Co., 516 F.Supp. 643, 656 (s.D.N.Y. 1981). Cfr. Columbia Broadcasting System, Inc. v. Stokely-Van Camp, Inc., 522 F.2d 369, 378 (2d Cir. 1975). No existió tal deber acá.

Los demandados igualmente fallaron en establecer la defensa basada en la caducidad. La parte que invoca la caducidad como defensa debe probar que la parte 
contraria "no ejerció sus derechos de manera diligente, y que tal falta de diligencia en el ejercicio [...] resultó en perjuicio para ellos". Lottie Joplin, 592 F.2d at 655, citando, inter alia, Costello v. United States, 365 U.S. 265, 282, 81 S.Ct. 534, 543, 5 L.Ed.2d 551 (1961). En Lottie Joplin, el Segundo Circuito sostuvo que un lapso aproximado de medio año entre la publicación del trabajo presuntamente infractor y la presentación de la demanda no configuraba una dilación suficiente para conceder la solicitud basada en la caducidad. En este caso, los demandados fueron informados durante semanas de la desaprobación del demandante frente a su póster; además, no presentaron evidencia que señalara que de haber tenido conocimiento de la reacción del demandante, hubieran modificado sus acciones subsiguientes. En consecuencia, fallaron en probar perjuicios causados a ellos.

VI.

Por las razones presentadas antes, el juicio sumario es concedido en favor del demandante en cuanto a la copia.

Se celebrará audiencia prejudicial el 11 de septiembre de 1987, a las 2 p. m. en punto, en la sala de audiencias 35 , para determinar la adecuada medida y reconocimiento de daños y perjuicios del caso, otros asuntos pertinentes, la agenda de los demandados y otros procedimientos posteriores. Las partes podrán reunirse con anterioridad a esta audiencia, y llegar a un acuerdo sobre estos particulares, si ello fuere posible. 